\title{
Percepción social sobre los beneficios de estar viviendo en un área natural protegida: Otoch Ma'ax Yetel Kooh
}

\author{
Wilian de Jesús Aguilar Cordero* \\ Campus de Ciencias Biológicas y Agropecuarias, \\ Universidad Autónoma de Yucatán \\ David E. Alonzo Parra \\ David Canul Rosado \\ Jorge Cerón Gómez \\ Dumack, A. C.
}

\section{Resumen}

El Área de Protección de Flora y Fauna (APFF) Otoch Ma’ax Yetel Kooh, ubicada en los límites de los estados de Yucatán y Quintana Roo, resulta un ejemplo de concientización de los residentes en la búsqueda de mejores condiciones de vida y la necesidad de conservar su entorno ecológico, ya que fueron las comunidades que están dentro del área quienes solicitaron su denominación como área natural protegida (ANP). El presente estudio aborda la percepción de las comunidades de la Reserva Otoch Ma'ax Yetel Kooh acerca de los beneficios tangibles e intangibles que obtienen de esta área protegida y su zona de influencia. El estudio se apoya en la metodología de la antropología, la observación directa, entrevistas y cuestionarios. De acuerdo con los resultados: 82 \% de la población encuestada tiene deseos de participar en nuevos proyectos de la Comisión Nacional de Áreas Naturales Protegidas (Conanp), aunque hay también puntos de vista que destacan la necesidad de fomentar y fortalecer el diálogo horizontal entre la comunidad y la Conanp.

\section{Palabras clave}

Otoch Ma’ax Yetel Kooh, beneficios tangibles e intangibles, percepción y ANP. 


\title{
Social perception of benefits of living in Otoch $\mathrm{Ma}^{\prime} \mathrm{ax}$ Yetel Kooh, a protected natural area
}

\author{
Wilian de Jesús Aguilar Cordero* \\ Campus de Ciencias Biológicas y Agropecuarias, \\ Universidad Autónoma de Yucatán \\ David E. Alonzo Parra \\ David Canul Rosado \\ Jorge Cerón Gómez
} Dumack, A. C.

\begin{abstract}
Otoch Ma'ax Yetel Kooh is a flora and fauna protected area, bordering the states of Yucatan and Quintana Roo. This is a clear example of local awareness by the communities themselves who, in their search for better living conditions and their need to preserve the ecological environment, requested the status as a natural protected area (ANP). This study addresses the perception of communities within Otoch Reserve Ma'axYetel Kooh on the tangible and intangible benefits they get from this protected area and its surroundings. The study is based on the methodology of anthropology, direct observation, interviews and questionnaires. According to the results $82 \%$ of the surveyed population has a desire to participate in new projects with the National Commission of Natural Protected Areas (Conanp), and there are also views that highlight the need to promote and strengthen horizontal dialogue between communities and Conanp.
\end{abstract}

\section{KEY WORDS}

Otoch Ma’ax Yetel Kooh, tangible and intangible benefits, perception and ANP. 


\section{Introducción}

En el contexto de la creación de áreas naturales protegidas (ANP) en el nivel nacional, es singular el caso del Área de Protección de Flora y Fauna (APFF) Otoch Ma'ax Yetel Kooh ("la casa del mono y el puma", en lengua maya), en los límites de los estados de Yucatán y Quintana Roo, dadas las circunstancias en que se originó, en el año 2002: las propias comunidades locales, al visualizar las características de su entorno silvestre, sus riesgos y sus potencialidades, solicitaron a los organismos de gobierno su declaración como ANP (Ramos-Fernández et al., 2004).

El APFF Otoch Ma'ax Yetel Kooh, cuya administración está a cargo de la Comisión Nacional de Áreas Naturales Protegidas (Conanp), conserva una muestra representativa de la selva subperenifolia, de la selva baja inundable y del pastizal inundable de tipo marisma, conocido como sabana, y su fauna asociada, que incluye numerosas especies en situación de compromiso poblacional en el ámbito nacional. Con una superficie de 5367 ha, el área resulta de gran relevancia cultural y arqueológica, con evidencias de ocupación humana que datan de 2500 a. C. Una importante población de monos araña (Ateles geoffroyi), un conjunto de lagunas, cenotes y cavernas, son algunos de los principales atractivos del lugar (Conanp, 2006).

Dentro del área protegida se localizan dos comunidades, Punta Laguna y Yodzonot, y en su zona de influencia nueve comunidades de menor tamaño, entre las que se cuentan Esmeralda, Campamento Hidalgo, Banco Cenote, Laguna Chabela, Chunyaxché, Santa Rufina, Santa Marta, La Ceiba y Guadalupe (Conanp, 2006). En todos los casos, se trata de comunidades de linaje maya, que conservan el uso de su lenguaje, sus modos de producción tradicionales, la tipología constructiva de sus viviendas, y sus ceremonias ancestrales.

Transcurridos ya varios años desde la creación del APFF, deviene oportuno indagar acerca de la percepción que tienen las comunidades locales sobre los beneficios (tangibles e intangibles) que les ha reportado el estatus de ANP que rige sobre "su" territorio. Se entiende como beneficios tangibles aquellos que pueden ser transformados en dinero líquido, ser intercambiados por productos o ser utilizados como alimento para el autoconsumo, mientras que los intangibles se definen como aquellos que proporcionan bienestar y que no pueden ser transformados en dinero, 
pero que afectan directamente el estado anímico o bien son empleados en el medio social para interaccionar con él (Vieyra et al., 2004).

Esta investigación surge de la solicitud de los miembros de la Dirección del APFF Otoch Ma'ax Yetel Kooh de saber qué piensa la gente sobre el desempeño de la Conanp. Con base en ello, se planteó como objetivo general conocer la percepción de tres comunidades acerca de los beneficios tangibles e intangibles que obtienen por vivir dentro del APFF Otoch Ma'ax Yetel Kooh, así como saber cuál es la percepción de las comunidades que habitan dentro y fuera de la reserva sobre el impacto de la creación y administración del área por parte de la Conanp.

\section{Marco teórico}

A principios de la década de los ochenta se creó en México el Sistema Nacional de Áreas Naturales Protegidas (Sinap), gracias a lo cual las experiencias de conservación en el país han aumentado. Si bien aún no se ha logrado la total participación social de los usuarios en la realización de los planes de manejo de tales áreas, los residentes de estos lugares han demostrado a lo largo de su historia la aplicación de una lógica propia y funcional en la utilización de los recursos naturales (Aguilar y Sosa, 2008).

En las últimas décadas, la relación áreas protegidas-comunidades ha evolucionado conceptualmente de manera considerable. Tras muchos años de aplicación de modelos de gestión netamente excluyentes, en los que comunidades y pobladores locales eran vistos como una amenaza para la conservación de los espacios silvestres protegidos, en la actualidad ha sido cada vez más evidente, para las instituciones de gobierno encargadas de administrar las ANP, la importancia de incorporar formalmente en su gestión a estos actores. Tal cambio de actitud no sólo obedece a una reivindicación de los derechos de las comunidades sobre determinados territorios y sobre el uso de sus recursos naturales; es a la vez un reconocimiento a sus prácticas ancestrales de bajo impacto ambiental, al conocimiento del entorno silvestre en que viven, y al aporte que en consecuencia pueden brindar para una adecuada gestión de las ANP.

El devenir histórico muestra que la creación de nuevas ANP ha sido casi siempre resultado de iniciativas gubernamentales, bajo el fundamento del bienestar general 
que conlleva la conservación del patrimonio cultural y natural de una región, estado o nación.

Hoy en día, uno de los principios rectores de toda política de gestión de ANP es que las comunidades locales participen activamente en la gestión de los espacios protegidos que las involucran, y sean los beneficiarios directos de sus bienes y servicios.

\section{Importancia de la participación social en el manejo de las ANP}

La participación social es un proceso por medio del cual los distintos actores se colocan en función de sus propios intereses, interviniendo, directamente y por medio de sus representantes, en la marcha de los diferentes aspectos de la vida colectiva. Significa la oportunidad de expresar de forma horizontal su opinión y tomar las decisiones pertinentes que, con base en su experiencia, afecten sus vidas y el contexto donde se desarrollan, para favorecer su participación en igualdad de condiciones (Sosa y Aguilar, 2000).

Las necesidades e intereses son contemplados para definir planes y proyectos, por lo que también son el referente para evaluar el impacto de las acciones emprendidas. En este proceso intervienen los sujetos y las instituciones involucradas, teniendo la posibilidad de transformar los hechos concretos. La participación de la sociedad en la toma de decisiones y evaluación de las políticas ambientales es una condición necesaria para dotar a éstas de legitimidad y generar condiciones propicias para su aplicación. Sin duda, la participación organizada de la sociedad constituye un requisito indispensable para transitar hacia niveles sostenibles de desarrollo, reconociendo el papel cardinal de los actores sociales en torno a la con-servación de los ecosistemas y lo imperativo de su colaboración en cualquier diseño para la planeación, el manejo y el aprovechamiento de los recursos naturales (Sosa y Aguilar, 2000).

Los instrumentos de política y lineamientos vigentes en cuanto al manejo de los recursos naturales reconocen que una de las dimensiones fundamentales (a la vez reto y fortaleza de la sustentabilidad) es la participación social para la gestión y manejo de los recursos naturales, en busca de estrategias de desarrollo culturalmente apropiadas y apropiables. Éstas deben ser capaces de mantener los procesos ecológicos esenciales para la conservación de la biodiversidad y, al mismo tiempo, elevar la calidad de vida de la sociedad. La noción de sustentabilidad ha sido acep- 
tada por la legislación ambiental mexicana, con base en los requerimientos concretos de acciones y políticas específicas que permitan revertir el proceso de deterioro ambiental (Benet, Soares y Setién, 1997).

Históricamente, la creación de áreas protegidas traía consigo una serie de conflictos sociales, ya que este proceso partía de la toma de decisiones de un sector, sin tener en cuenta a la población que habitaba en las áreas. En ese sentido, se emprendía la expulsión de la población local de las nuevas áreas protegidas, propiciando conflictos por el uso y manejo de los recursos naturales. Sin embargo, en los últimos años, la participación social en el manejo de las ANP se ha convertido en una herramienta de gestión que, acompañada de otras estrategias como la educación ambiental, la formación y capacitación en procesos productivos sostenibles, la comunicación, el ordenamiento territorial, la creación de unidades de manejo sustentable de vida silvestre, y algunas más, ha generado conocimiento y cambios de actitudes y prácticas en relación con la percepción biocultural ${ }^{1}$ de las áreas protegidas de América Latina. No obstante, todavía queda un largo camino por recorrer, ya que los procesos de participación social no siempre son una realidad, aun quedando explícitos en la formulación de los programas de manejo de las ANP, lo cual no significa su desarrollo e implementación.

Cuando la normatividad y regulación formal de un ANP es aplicada vertical y autoritariamente, puede que los campesinos y/o indígenas sean tratados como "delincuentes ambientales" en su propio territorio, el cual han manejado y aprovechado desde épocas pretéritas, y lo siguen haciendo en la actualidad, al constituir una estrategia de sobrevivencia para la producción y reproducción de las familias campesinas pobres que "desafortunadamente" viven dentro de un ANP (Aguilar y Núñez, 2009).

${ }^{1}$ Aunque la percepción ha sido concebida como un proceso cognitivo, estamos de acuerdo con Vargas Melgarejo (1994: 47), quien señala que "la percepción es biocultural porque, por un lado, depende de los estímulos físicos y sensaciones involucrados y, por otro lado, de la selección y organización de dichos estímulos y sensaciones. Las experiencias sensoriales se interpretan y adquieren significado moldeadas por pautas culturales e ideológicas específicas aprendidas desde la infancia”. 


\section{Materiales y métodos}

\section{Área de estudio}

El área de estudio está localizada en el APFF Otoch Ma'ax Yetel Kooh, situada al noreste de la Península de Yucatán, a $18 \mathrm{~km}$ al norte de la zona arqueológica de Cobá

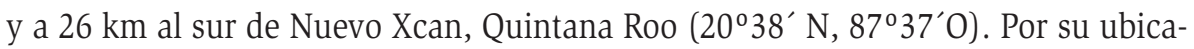
ción geográfica, el APFF se sitúa dentro de los límites del estado de Quintana Roo, pero administrativamente pertenece al ejido de Valladolid, en el estado de Yucatán.

Dentro del APFF se encuentran las comunidades de Kankabchen, Punta Laguna y Yodzonot. En la zona de influencia del área se ubican pequeñas poblaciones conocidas como Campamento Hidalgo, Banco Cenote, Laguna Chabela, Chunyaxché, La Ceiba, Santa Marta, Santa Rufina, Guadalupe y Laguna Esmeralda (figura 1).

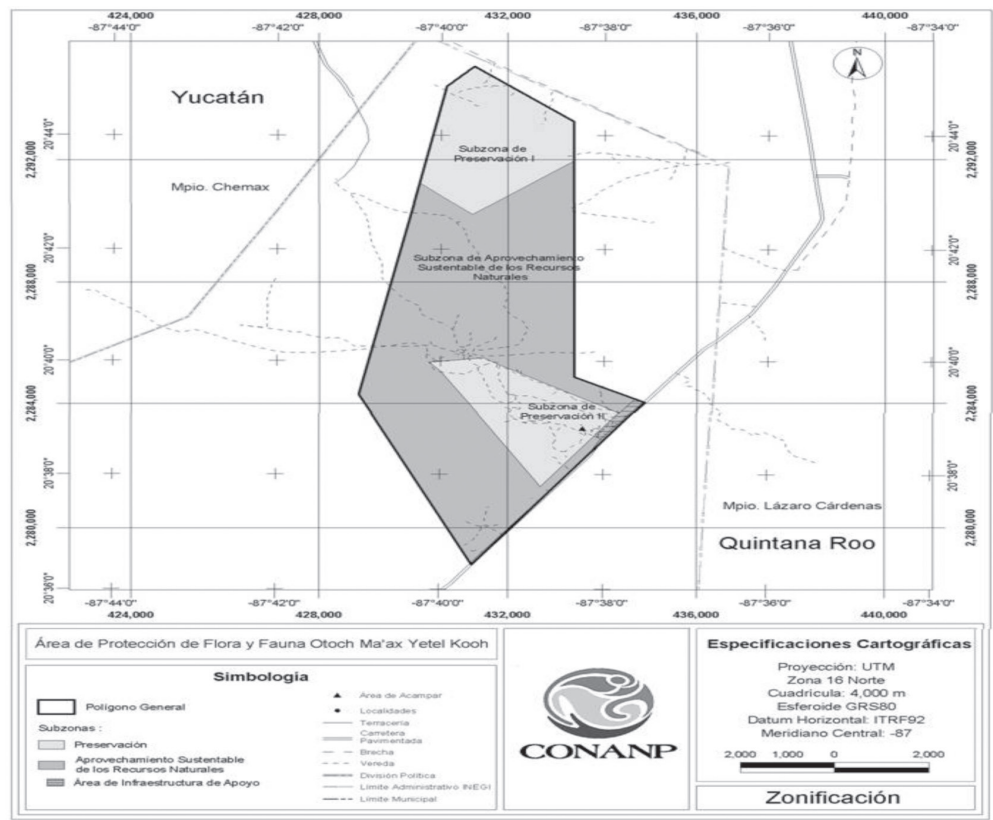

Fuente: Conanp, 2006. 
Se trabajó con dos de las comunidades asentadas dentro del APFF, Punta Laguna y Yodzonot, así como con la comunidad Esmeralda, la cual se encuentra en el área de influencia.

\section{COMUnidAd DE PUnTA LAGUnA}

Está inmersa en el APFF, situada al noreste de la Península de Yucatán, $18 \mathrm{~km}$ al norte de la zona arqueológica de Cobá y $26 \mathrm{~km}$ al sur de Nuevo Xcan. Según el Programa de Conservación y Manejo del APFF vigente, en esta comunidad viven 22 familias, con un total de 95 habitantes; 58 \% de la población es bilingüe (habla maya y español) y el resto sólo habla maya. La mayoría de las casas son típicamente mayas -construidas con materiales tradicionales como palma de huano (Sabal yара), tierra, madera, etc.-- Poseen agua potable y energía eléctrica a través de paneles solares.

Las familias fundadoras de esta comunidad tomaron la iniciativa para conservar el área. En 1979 se iniciaron las acciones de protección y conservación por parte de las instituciones gubernamentales, cuando el Instituto Nacional de Antropología e Historia (INAH) facultó al señor Serapio Canul (uno de los fundadores de Punta Laguna, oriundo de Chemax) como "custodio" del sitio arqueológico (Conanp, 2006).

En 1994, los pobladores de la comunidad comenzaron las gestiones para nombrar el área Santuario Natural del Mono Araña. La presencia de esta especie constituye un recurso sustancial para los habitantes desde el punto de vista económico, a través del turismo de intereses especiales. Actualmente están organizados y constituidos formalmente como Sociedad Cooperativa Najil Tucha (Conanp, 2006).

El Instituto Nacional de Estadística y Geografía (INEGI) reportó para el año 2000 una población económicamente activa de 26 personas, 21 ocupadas en el sector primario y cinco en el sector secundario (Conanp, 2006).

\section{YODZONOT}

Se localiza dentro del ANP y se llega a ella por dos senderos $(4 \mathrm{~km})$ que comunican con la carretera Cobá-Nuevo Xcan. Está conformada por ocho familias con un total de 25 habitantes, de los cuales $52 \%$ son adultos (siete hombres y seis mujeres) 
y $48 \%$ jóvenes y niños; $52 \%$ son hombres y $48 \%$ mujeres. Últimamente se ha observado una disminución de sus pobladores debido a la migración hacia otras localidades más cercanas a la carretera Cobá-Nuevo Xcan en busca de trabajos más redituables. Además del comisario ejidal, muy pocas personas hablan español. El grado promedio de escolaridad es $3^{\circ}$ de primaria. Todas las casas están fabricadas de barro, piedra, madera y huano, al estilo maya. En cuanto a los servicios, se cuenta sólo con un sistema de paneles solares para abastecer ocho casas. Por medio de una bomba de mecate (de fuerza de empuje), ubicada sobre un cenote en el centro de la comunidad, se suministra el agua. Sus principales actividades productivas son la agricultura y la apicultura (Conanp, 2006).

\section{COMUNIDAD ESMERALDA}

Está ubicada a $4 \mathrm{~km}$ de la población Tres Reyes, sobre la carretera Cobá-Nuevo Xcan, a pocos kilómetros de Punta Laguna. En 2000, el INEGI reportó que su población total era de 24 habitantes, de los cuales 14 son hombres y diez mujeres; 11 son mayores de 18 años, y de éstos, diez hablan solamente maya y uno maya y español. Existen cuatro hogares; todas las casas están construidas al estilo maya (de piedras y madera); ninguna cuenta con material de desecho ni láminas de cartón. El grado promedio de escolaridad es hasta $3^{\circ}$ de primaria. La población económicamente activa la conforman cuatro personas que trabajan en el sector primario. Respecto a los servicios, ninguna vivienda tiene agua entubada, drenaje ni energía eléctrica, y ningún habitante cuenta con radio, televisión ni automóvil. Las principales actividades económicas son la milpa, la caza, la apicultura, la producción de carbón, la crianza de borregos y la producción de leña (milpa y selva) (Conanp, 2006).

\section{Métodos de recolección de datos}

Con el propósito de conocer el área de estudio, se hizo un recorrido inicial en las comunidades con el fin de familiarizarse, así como de contactar a las autoridades formales para notificarles de la investigación que se realizaría. El trabajo de campo se llevó a cabo durante noviembre de 2010. 
Para obtener los datos de campo, se diseñó un cuestionario mixto de 26 preguntas mediante el cual se buscó saber si los pobladores conocen el plan de manejo y qué es la Conanp, si se les tomó en cuenta en la elaboración del plan de manejo, cómo es su relación con la administración de la reserva (buena, regular o mala), qué beneficios pueden tener sobre los servicios ambientales y/o el manejo y aprovechamiento de los recursos naturales, y si consideran importante fortalecer los vínculos de colaboración con la dirección de la reserva. El cuestionario fue aplicado a una muestra al azar de 29 hogares (mayores de edad), integrando a dos de las comunidades estudiadas: Punta Laguna $(\mathrm{N}=21)$ y Esmeralda $(\mathrm{N}=8)$.

El método cualitativo se basó en la aplicación de ocho entrevistas informales a gente de las tres comunidades; éstas se fundamentaron en la discusión previa del grupo de investigación sobre algunas preguntas clave, por ejemplo, ¿cómo influye en su modo de vida estar dentro y cerca de un ANP? (conocimiento general de qué es un ANP, beneficios y desventajas), ¿sabe quién está a cargo del ANP? (conocimiento sobre la existencia de la Conanp), ¿ha participado en algún(alguna) proyecto/ curso/capacitación? (conocimiento sobre las funciones de la Conanp) y ¿de qué manera le han servido estos(as) proyectos/cursos/capacitaciones? (transformación y autogestión de los sistemas de producción a partir del trabajo realizado por la Conanp). Estos interrogantes fueron el eje central para efectuar las entrevistas informales.

En Yodzonot, dado que sólo tres familias se encontraban en sus casas, se decidió realizar las entrevistas y no los cuestionarios. De las cinco entrevistas restantes, cuatro fueron en Punta Laguna y una en Esmeralda.

Finalmente, otra técnica utilizada en el trabajo de campo fue la observación ordinaria, que permitió recoger información numerosa, directa, rica, profunda y compleja, con el apoyo de una guía de observación, haciendo recorridos por las tres comunidades para prestar atención a las características de la vivienda, la vestimenta y las actividades productivas, entre otros aspectos (Sánchez, 2001; Aguilar, 2000).

El trabajo de gabinete consistió en capturar los datos de los cuestionarios en una base de datos, previamente elaborada en una tabla de Excel, y posteriormente procesarlos, analizarlos y presentarlos en gráficas, segregando los porcentajes en cada uno de los componentes. Los datos de las entrevistas y la observación fueron transcritos y analizados para su inclusión en los resultados. 


\section{Resultados y discusión}

\section{Percepción social de Punta Laguna y Yodzonot sobre los beneficios tan- gibles e intangibles}

El análisis de los datos obtenidos de las entrevistas y cuestionarios aplicados a moradores de Punta Laguna refleja que éstos tienen conocimiento tanto de la existencia de un ANP en su comunidad como de sus implicaciones. Esto incluye la delimitación y en algunos casos la reducción del espacio geográfico y la eliminación de algunas prácticas tradicionales. El uso de los recursos naturales ahora se limita a un aprovechamiento regulado no extractivo, y la milpa se ha visto reducida debido a los riesgos implícitos de incendios forestales y tala, práctica que (según dos entrevistados) se sigue realizando en aquellos sectores donde la fiscalización es inexistente.

La comunidad reconoce que la conservación de estos espacios genera beneficios tangibles e intangibles; los beneficios tangibles que resalta son:

- Mayor conectividad del área (reflejada en estructura vial), que acarrea múltiples beneficios en términos de menor aislamiento

- Mayor empleo generado por el turismo de intereses especiales -observación de fauna silvestre y especialmente de aves (birdwatching), ceremonias mayas y otras manifestaciones de ecoturismo-, actividad que ha crecido gracias a una mayor difusión del área por su estatus de APFF

- Aprovechamiento de líneas de financiamiento gubernamentales y de otros tipos, a través de proyectos de artesanía, turismo, apicultura, etc., de manera que destaca la adquisición de equipamiento para ecoturismo, la construcción de cabañas, el uso de paneles solares como fuente de energía eléctrica, etcétera

Por otro lado, la comunidad menciona como beneficios no cuantificables (intangibles) la conservación de flora y fauna, la sombra, la moderación del microclima y la captación de carbono, entre otros servicios (gráfica 1). 


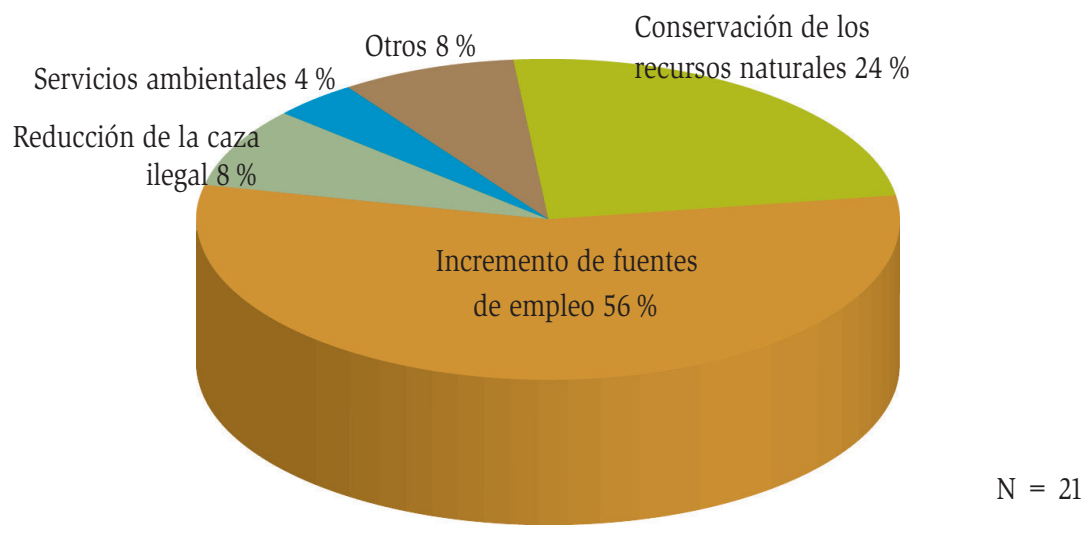

Fuente: elaboración propia, 2010.

Grafica 1. Beneficio que el APFF Otoch Ma'ax Yetel Kooch provee, según la comunidad de Punta Laguna, Yucatán

Así pues, la respuesta de la comunidad es favorable ( $82 \%$ ) a participar en nuevos proyectos de la Conanp. Además, la comunidad colabora en el cuidado de los recursos naturales del ANP, principalmente en actividades de vigilancia, al inspeccionar que no se realicen actividades prohibidas por el programa de manejo del APFF y por sus propias normas, y que también las labores de investigación se lleven a cabo con apego al programa. Los entrevistados indicaron ser promotores de la regulación del ANP con el ejemplo.

Cabe apuntar que una minoría de los entrevistados señaló su desacuerdo con la manera en que la Conanp lleva los proyectos, debido a que (según ellos) los montos que les informan en un comienzo del proceso no coinciden con los entregados efectivamente. En este sentido, sugieren mayor comunicación para que ellos comprendan bien lo sucedido.

En el caso de la comunidad de Yodzonot, su principal fuente de ingresos es un proyecto apícola que durante las épocas de primavera y verano genera 900 kilos de miel producida por las abejas africanizadas (Apis mellifera). Actualmente está comenzando el proceso para cosechar miel real, que es cotizada a un mejor precio en el mercado, y se cosecha miel de las abejas Melipona, la cual es cotizada a un precio más alto porque el frasco de 200 g tiene un valor en el mercado de 400 pesos. 
En esta comunidad también existe un proyecto que está iniciando: una Unidad de Manejo para la Conservación de la Vida Silvestre (UMA) de venados, los cuales servirán para reproducción y, el producto de éstos, para alimentación de los pobladores 0 , en su caso, para venta y generación de ingresos.

Las mujeres han sido capacitadas en proyectos de huertos familiares y composta, para que en su mismo espacio familiar cuenten con recursos alimenticios y no tengan que caminar tanto, ya que el acceso a la comunidad es difícil y con este proyecto tienen a la mano los ingredientes necesarios para la preparación de los alimentos.

Es interesante mencionar que dentro de esta comunidad prevalece un sistema amigable con el ambiente: de captación de energía solar por medio de paneles que generan energía para las viviendas y la bodega donde se guardan los insumos utilizados en el proyecto de la miel, y hay un refrigerador que ayuda a preservar los alimentos en buen estado. Asimismo, se ha aplicado un proyecto de cocinas ahorradoras de leña, con el cual se previenen algunos problemas de salud y a la vez se beneficia al bosque porque disminuye la tala de árboles.

La presencia de la Conanp ha sido muy bien valorada por los pobladores de Yodzonot, pues desde su llegada ha brindado apoyo, llevando los proyectos antes mencionados acompañados de sus respectivas capacitaciones para lograr un mejor desarrollo.

Desde el punto de vista de los habitantes, una de las necesidades más urgentes es la construcción de una calle que les permita transitar con mayor libertad, porque el acceso hacia la comunidad es a pie y son dos kilómetros aproximadamente los que hay que caminar para llegar a ella, además de que esto también dificulta la comercialización de sus productos.

\section{La visión de los de afuera: el área de influencia, la comunidad de Esmeralda: beneficios tangibles e intangibles}

Todos los encuestados de la comunidad de Esmeralda $(\mathrm{N}=8)$ están enterados de que Otoch Ma'ax Yetel Kooh es un ANP, la cual, de acuerdo con su percepción, fue creada principalmente para la conservación de los animales que allí habitan. Conciben esta área como un espacio físico alejado geográficamente de su comunidad, por 
lo tanto no sienten que les pertenezca ni que les beneficie o perjudique su existencia. Por el contrario, tienen la idea de que el ANP pertenece a las comunidades que están cerca de ella (Punta Laguna y Yodzonot). Esta apreciación se hizo evidente al preguntarles su opinión sobre el área, ya que su primera reacción fue expresar que no está cerca de su comunidad y que desconocen tanto al personal de la Conanp como sus actividades. Tampoco saben los motivos por los cuales fue creada el área y cómo ésta es regulada a través de un programa de manejo. Este desconocimiento puede partir del hecho de que, según lo expresado, la comunidad Esmeralda no fue tomada en cuenta en la creación de la reserva ni ha sido beneficiada con los programas que ofrece la Conanp. En consecuencia, al no tener mayores elementos que permitiesen profundizar sobre la visión de la comunidad en torno a la reserva y sus beneficios tangibles e intangibles, las entrevistas estuvieron más bien dirigidas a identificar cómo ésta percibe y maneja su territorio.

En general, la comunidad Esmeralda tiene presente la importancia de conservar sus valores naturales, primordialmente los animales y el cenote que está localizado en el interior de la misma, ya que sus actividades de conservación están enfocadas al mantenimiento del cenote y al cuidado de sus plantas y animales, reconociendo que preservar sus valores naturales y culturales constituye un potencial turístico.

A lo largo de las entrevistas informales se tuvo conocimiento de que, durante 2009, la comunidad fue beneficiada mediante una empresa privada de ecoturismo (Alltournative), que movió la economía local al generar trabajo directo e indirecto, como la venta de artesanías y alimentos y el uso del cenote. Pero también de que el turismo experimentó un decrecimiento total, debido a la posible epidemia de influenza que amenazaba el país, lo cual trajo consigo que la empresa se retirara de la comunidad y no se continuara desarrollando la actividad turística, como declara un entrevistado:

ya no iba turismo a la comunidad y tiempo después regresó la empresa al poblado por el equipo para hacer dichas actividades y nunca regresaron, nos dijeron que venían por el equipo para cambiarlo por uno más nuevo, y se lo llevaron y nunca más lo trajeron de vuelta, parece que nos abandonaron [Desiderio, entrevista personal, 10 de octubre de 2010].

Esta situación hace suponer la poca capacidad de autogestión de la comunidad, la cual podría verse fortalecida mediante los programas que promueve la Conanp. 
La ausencia de opciones laborales ha orillado a los pobladores a dedicarse únicamente al manejo de sus tierras, lo que constituye su principal ingreso económico.

A pesar de las dificultades, los avances y los retos a los que se enfrentan a diario las comunidades humanas asentadas en la reserva, y de la necesidad de apropiación de los recursos naturales de manera sustentable, éstas requieren afianzar los vínculos de colaboración con las autoridades ambientales, así como conocer las principales propuestas de manejo y aprovechamiento de los recursos naturales que puedan mejorar sus condiciones de vida y consolidar su inclusión social. En este tenor, García Frapolli y Toledo (2008: 104) plantean que "si la gestión de estas ANP está bien llevada a cabo, las comunidades que habitan en ellas tienden a participar activamente en el manejo de estos territorios".

\section{A manera de reflexión final}

Con base en los 29 cuestionarios aplicados y la elaboración de ocho entrevistas se concluyó que las tres comunidades estudiadas conocen el ANP, pero sólo las dos comunidades asentadas en ella participaron activamente en su creación. Asimismo, estas dos comunidades se han beneficiado en mayor medida de los valores tangibles e intangibles del área, ya que han tenido acceso a capacitación y a apoyos gubernamentales debido a su localización. Por el contrario, para la comunidad Esmeralda la creación del área prácticamente ha sido indiferente, ya que además de no haber participado en ese proceso, no se ha beneficiado de ella.

En términos generales, $87 \%$ de los encuestados encuentra positivo el establecimiento de la reserva, ya que la consideran un lugar de protección para plantas y animales. Los encuestados de Punta Laguna y Yodzonot reconocieron que hay un incremento de las fuentes de empleo relacionadas directa o indirectamente con el aumento de la actividad turística. Además, se han implementado proyectos alternos (apicultura, carbón, artesanía y otros productos del Programa de Empleo Temporal -PET-, el Programa de Conservación para el Desarrollo Sostenible -Procodes-, y otros proyectos ejecutados por la Conanp y la Procuraduría Federal de Protección al Ambiente -Profepa-). Para la comunidad Esmeralda, uno de los mayores beneficios de la creación del ANP fue la generación de fuentes de trabajo, ya que una empresa interesada en el ecoturismo construyó infraestructura y prestó equipos, promoviendo empleo directo e indirecto. No obstante, por múltiples factores disminuyó el 
turismo y la empresa se retiró del lugar, dejando a los pobladores sin posibilidad de continuar esta actividad.

En cuanto a las percepciones negativas, las comunidades de Punta Laguna y Yodzonot admiten que el ANP ha causado restricciones a sus actividades tradicionales como la caza y la tala de árboles, además de provocar la eliminación de la práctica agrícola tradicional y la reubicación de las familias, con una inmediata reducción de los espacios y alternativas de sustento, aunque el área ya tenía un estatus de protección desde 1982. En la comunidad Esmeralda prácticamente no identifican que el establecimiento de la reserva haya traído consecuencias negativas, pero $14 \%$ de la población sí subraya la prohibición de producir milpa en el área y el hecho de no haber sido considerados en la creación y gestión del ANP.

En Punta Laguna y Yodzonot, los principales medios por los cuales se han enterado de las actividades de la Dirección de la reserva han sido las pláticas con el personal del lugar y los folletos y carteles. Por su parte, las conversaciones entre los habitantes de la comunidad han servido para dar a conocer las actividades de la reserva.

De los encuestados en Punta Laguna, 77 \% indica que la cooperativa Najil Tucha está en condiciones de dar seguimiento a los proyectos por su cuenta con asesorías de organizaciones no gubernamentales, y así los beneficios obtenidos llegarían íntegros y directamente a la cooperativa. En Yodzonot, el total de los encuestados expresa estar de acuerdo en realizar las acciones o proyectos, ya que representan un beneficio para ellos, como el mejoramiento de sus ingresos económicos. En ambas comunidades, aproximadamente la mitad de los encuestados conoce los nuevos proyectos, y casi todos están interesados en participar en nuevos proyectos.

Es importante remarcar que el conocimiento de la existencia del programa de manejo del área en Yodzonot es de 100 \% y en Punta Laguna de 78 \%. Estos porcentajes son resultado de la constante interacción de la Conanp con estas comunidades, sin embargo la encuesta no permite evaluar el grado en que se conoce el plan de manejo. Por otro lado, en la comunidad Esmeralda, los encuestados mencionan una aparente falta de presencia institucional de la Conanp, lo cual podría ser reflejo del menor conocimiento del programa de manejo, por lo que $75 \%$ de los encuestados no está al tanto de los lineamientos establecidos para el uso del área protegida.

En lo que respecta a las comunidades asentadas en el interior del ANP, se observó una buena percepción de la interacción entre la comunidad y la Conanp. Asimismo, la reserva es percibida como un bien común que, con un adecuado cuidado, 
constituye una fuente de ingresos y sustento familiar, al existir una mayor oferta laboral vinculada principalmente a la actividad turística (visitas guiadas, venta de artesanías, capacitación, etcétera).

Conseguir que la protección del área fuera de carácter federal representa un logro que por muchos años fue gestionado por los habitantes de la comunidad de Punta Laguna, con objeto de garantizar la legal conservación de los recursos naturales y beneficiarse de ello.

Cabe destacar que en Yodzonot y Esmeralda fue notoria la carencia de servicios básicos como electricidad, transporte, servicio médico y escuela. De tal modo, es fundamental establecer lazos interinstitucionales para favorecer a los habitantes de estas localidades por igual, siguiendo las estrategias planteadas en el programa de manejo del ANP.

\section{FUENTES CONSULTADAS}

Aguilar, C. W. (2000). "Relevancia de los aspectos socioculturales en la conservación y el manejo de la vida silvestre”. En O. Sánchez, M. C. Donovarros-Aguilar y J. E. Sosa Escalante (eds.). Conservación y manejo de vertebrados en el trópico de México. Diplomado en Conservación, Manejo y Aprovechamiento de Vida Silvestre. México: Unidos para la Conservación/Agrupación Sierra Madre/Secretaría de Medio Ambiente, Recursos Naturales y Pesca (Semarnap), 35-46.

Aguilar, C. W. y D. E. Núñez (2009). "Cultura, identidad y estrategias de sobrevivencia de los campesinos pobres de un área natural protegida: la reserva municipal de Cuxtal del Municipio de Mérida, Yucatán, México". En Memoria del III Congreso Internacional de Antropología Social desde la Frontera Sur. Chetumal: Universidad de Quintana Roo, 327-342.

Aguilar, C. W. y E. J. Sosa (2008). "Percepción, conflicto y propuestas de participación social en torno a la conservación de la biodiversidad de la Reserva de la Biosfera Ría Lagartos, Yucatán, México”. En C. Serrano Sánchez y M. Cardoso Gómez (eds.). El Mediterráneo americano: población, cultura e historia. En homenaje a Don Antonio Pompa y Pompa. XXVII Mesa Redonda de la Sociedad Mexicana de Antropología. Vol. II. México: Instituto de Investigaciones Antropológicas-Universidad Nacional Autónoma de México/Instituto Nacio- 
nal de Antropología e Historia/Sociedad Mexicana de Antropología, 797-811.

Benet, R., D. Soares y M. Setién (1997). Necesidad de una estrategia de participación social en las ANP’s de México [en línea]. México. Disponible en: http://www. era-mx.org/documentosinteres/manejosostenible/partsoc2.html [1997, 27 de febrero].

Conanp (2006). Programa de Conservación y Manejo Área de Protección de Flora y Fauna Otoch Ma’ax Yetel Kooh. México: Comisión Nacional de Áreas Naturales Protegidas.

Delgado, J. M. y J. Gutiérrez (1994). Métodos y técnicas cualitativas de investigación en ciencias sociales. Madrid: Síntesis.

García Frapolli, E. y V. M. Toledo (2008). "Evaluación de sistemas socioecológicos en áreas protegidas: un instrumento desde la economía ecológica”. Argumentos, 21 (56), 103-116.

Ramos-Fernández, G., et al. (2004). “El Área de Protección de Flora y Fauna Otoch Ma’ax Yetel Kooh: una iniciativa comunitaria de conservación de la biodiversidad”. VI Congreso Nacional de Áreas Naturales Protegidas. Monterrey, México.

Sánchez, S. R. (2001). “La observación participante como escenario y configuración de la diversidad de significados”. En M. L. Tarrés (coord.). Observar, escuchar y comprender. Sobre la tradición cualitativa en la investigación social. México: Miguel Ángel Porrúa/El Colegio de México/Facultad Latinoamericana de Ciencias Sociales, 97-131.

Sosa, E. J. y C. W. Aguilar (2000). Informe final del Diagnóstico de Participación Social de la Reserva de la Biosfera Ría Lagartos. México: Instituto Nacional Indigenista/Semarnap/Fondo Mexicano para la Conservación de la Naturaleza.

Vargas Melgarejo, L. M. (1994). “Sobre el concepto de percepción”. Alteridades, 4 (8), 47-53.

Vieyra, J., et al. (2004). "La participación de la mujer en la producción traspatio y sus beneficios tangibles e intangibles”. Cuadernos de Desarrollo Rural, 53, 9-23. 